\title{
The usefulness of preoperative MRI in synchronous bilateral breast cancer
}

\author{
Kosaka $Y^{1 \# *}$, Kikuchi $M^{1 *}$, Sengoku N1, Tanaka $Y^{1}$, Waraya $M^{1}$, Katoh $H^{1}$, Yamashita $K^{2}$, Kajita $S^{3}$, Woodhams $R^{4}$ and $W_{a t a n a b e ~} M^{5}$ \\ ${ }^{1}$ Department of Breast and Endocrine Surgery, Kitasato University School of Medicine, Kanagawa, Japan \\ ${ }^{2}$ Department of Upper Gastrointestinal Surgery, Kitasato University School of Medicine, Kanagawa, Japan \\ ${ }^{3}$ Department of Pathology, Kitasato University School of Medicine, Kanagawa, Japan \\ ${ }^{4}$ Department of Diagnostic Radiology, Kitasato University School of Medicine, Kanagawa, Japan \\ ${ }^{5}$ Department of Colorectal Surgery, Kitasato University School of Medicine, Kanagawa, Japan \\ \#Both authors contributed equally to this work
}

\begin{abstract}
Background: The incidence of synchronous bilateral breast cancer is increasing each year. The applicability of contrast-enhanced magnetic resonance imaging (MRI) in the detection of multiple breast lesions has been reported in many studies. However, there have been no reports concerning the usefulness of preoperative MRI to evaluate synchronous bilateral breast cancer in Japan. We reviewed patients with synchronous bilateral breast cancer to investigate the applicability of contrastenhanced MRI in detecting contralateral breast cancer that was not visible by MMG or ultrasound.

Methods: Synchronous bilateral breast cancer was found in 47 (3.2\%) of the 1465 breast cancer patients who underwent surgery in our hospital between April 2006 and December 2012. Of those 47 patients, we enrolled 28 patients whose second lesions were non-palpable. The sensitivities of MMG, ultrasonography, and contrastenhanced MRI were compared for their ability to detect the malignancy of the second lesion. We also assessed the pathological characteristics of those lesions that were only visible by contrast-enhanced MRI.
\end{abstract}

Results: In 28 patients whose second lesions were non-palpable,7\% was DCIS in first lesions, and 39\% was DCIS in second lesions. The median size of the invasive cancer was $2.3 \mathrm{~cm}$ (range, $0.5-9.5 \mathrm{~cm}$ ) within the first lesions. The median size of the invasive cancer was $1.0 \mathrm{~cm}(\mathrm{range}, 0.4-5.0 \mathrm{~cm})$ within the second lesions.

Of the 28 malignant lesions, 13 (46\%) were positive by MMG, 18 (64\%) were positive by ultrasonography, and 28 (100\%) were positive by contrast-enhanced MRI. In six of the 28 patients (21\%), the malignant lesions were found by contrast-enhanced MRI but not by MMG nor by ultrasonography.

Conclusion: Contrast-enhanced MRI has the highest sensitivity and is therefore the most useful modality in identifying non-palpable breast abnormalities. It is especially useful in the detection of small contralateral lesions and lesions immediately under the nipple of the contralateral breast.

\section{Introduction}

The incidence of synchronous bilateral breast cancer has been reported to be between $0.7 \%$ and $3.2 \%$ [1-3], which has increased each year in Japan. Specifically, the incidence was 3.5\% in 2004, $4.2 \%$ in 2007, and $5 \%$ in 2010, according to The Japanese Breast Cancer Society's Investigative Report on Registration of Breast Cancer Patients in Japan. Contrast-enhanced magnetic resonance imaging (MRI) has been demonstrated to be helpful for breast oncologists to accurately predict the extent of malignant lesions [4-6]. It has been reported that contrastenhanced magnetic resonance imaging (MRI) has high diagnostic ability for mass lesions with sensitivity and specificity of $99 \%$ and $89 \%$, respectively[7]. Compared to other countries, contrast-enhanced MRI tends to be used more frequently in Japan to ensure the safety of breast-conserving surgery. Therefore, it is possible that the widespread use of contrast-enhanced MRI in Japan has resulted in the increased incidence of synchronous bilateral breast cancer. However, there have been no reports concerning the usefulness of preoperative MRI to evaluate synchronous bilateral breast cancer in Japan. We reviewed data of patients with synchronous bilateral breast cancer to evaluate the usefulness of contrast-enhanced MRI in detecting contralateral breast cancer that was not visible by MMG nor by ultrasound. We then analyzed the pathological characteristics of tumors that were only visible by contrast-enhanced MRI.

\section{Material and method}

\section{Patients}

Synchronous bilateral breast cancer was found in 47 (3.2\%) of the 1465 breast cancer patients who underwent surgery in our hospital between April 2006 and December 2012. Of those 47 patients, 28 patients with non-palpable second lesions who underwent MMG, ultrasonography, and contrast-enhanced MRI before surgery were enrolled. In our study, the tumor that was first diagnosed is designated as the first lesion and its contralateral tumor is designated as the second lesion. MMG and ultrasonographic images used in the study were all obtained prior to performing contrast-enhanced MRI imaging. Patients ranged between 42 and 75 years of age (median, 66 years).

Correspondence to: Yoshimasa Kosaka, Department of Surgery, School of Medicine, Kitasato University, 1-15-1 Kitasato, Minami-ku, Sagamihara, Kanagawa, Japan; Tel: +81 427788111, Fax: +81 427789556; E-mail: y-kosaka@med.kitasato-u.ac.jp

Key words: synchronous bilateral breast cancer, MRI, Bilateral imaging

Received: November 28, 2017; Accepted: December 19, 2017; Published: December 23, 2017 


\section{Methods}

MRIs were performed on the patients in the prone position.

Bilateral breast images were captured 2-3 times on a gadoliniumenhanced dynamic MRI machine (GE Medical Systems, Milwaukee, Wis) with a dedicated bilateral breast coil.

Tumor stage, subtypes, and histopathological findings were compared between the first and the second lesions. The findings by mammography, ultrasonography, and contrast-enhanced MRI were then examined to see if they correctly identified the second lesions. The sensitivities of these modalities in detecting malignancies in the contralateral breast were also determined. The second lesions that were visible only by contrast-enhanced MRI were further reviewed for pathological characteristics, and we examined why MMG and US could not diagnose them. Furthermore, we investigated the occurrence of multiple lesions and family history in 28 cases. A case with two or more masses was defined as multiple lesions. Family history is defined as cases where breast cancer patients are present in first-degree relatives.

\section{Result}

\section{Tumor stage}

The TNM classification system was used for tumor staging. Table 1 lists a comparison of tumor stages between the first and the second lesions. With regards to the first lesion, two patients (7\%) were classified as Stage 0, 11 patients (39\%) were classified as Stage I, eight patients (29\%) were classified as Stage IIA, five patients (18\%) were classified as Stage IIB, one patient (3\%) was classified as Stage IIIA, and one patient (3\%) was classified as Stage IIIB. As for the second lesion, 11 patients (39\%) were classified as Stage 0, 12 patients (43\%) were classified as Stage I, three patients (11\%) were classified as Stage IIA, one patient (3\%) was classified as Stage IIB, and one patient (3\%) was classified as Stage IIIB.

\section{Hormone receptor and HER2 status}

Table 2 compares the hormone receptor and HER2 statuses between the first and the second lesions. Data regarding the ER status indicate that 23 patients $(82 \%)$ and 5 patients (18\%) had ER-positive and ER-negative first lesions, respectively. A total of 22 patients (79\%) and six patients (21\%) had ER-positive and ER-negative second lesions, respectively. With regards to HER2 status, three patients (11\%) had HER2-positive and 25 patients (89\%) had HER2-negative first lesions. No patient had HER2-positive second lesions, and thus all the second lesions were HER2-negative.

\section{Histopathological diagnosis}

The histopathological diagnoses for the first and second lesions are shown in Table 3. Within the first lesions, invasive ductal carcinoma (IDCa), ductal carcinoma in situ (DCIS), and mucinous carcinoma were found in $24(86 \%), 2$ (7\%), and 2 (7\%) patients, respectively. The median size of the invasive cancer was $2.3 \mathrm{~cm}$ (range, $0.5-9.5 \mathrm{~cm}$ ) within the first lesions. Within the second lesions, IDCa, DCIS, and mucinous carcinoma was found in 15 (54\%), 11 (39\%), and 2 (7\%) patients, respectively. The median size of the invasive cancer was 1.0 $\mathrm{cm}$ (range, $0.4-5.0 \mathrm{~cm}$ ) within the second lesions.

\section{Sensitivity for detecting malignancy of the second lesion}

Table 4 summarizes the sensitivities of MMG, ultrasonography, and contrast-enhanced MRI in detecting the malignancy of the second lesion. Contrast-enhanced MRI identified lesions in all of the 28 patients
Table 1. Tumor stages of the first and second lesions.

\begin{tabular}{|c|c|c|}
\hline & First lesion & Second lesion \\
\hline & n. (\%) & n. (\%) \\
\hline stage0 & $2(7)$ & $11(39)$ \\
\hline stage $\square$ & $11(39)$ & $12(43)$ \\
\hline stage $\square$ A & $8(29)$ & $3(11)$ \\
\hline stage $\square$ B & $5(18)$ & $1(3)$ \\
\hline stage $\square$ A & $1(3)$ & 0 \\
\hline stage $\square$ B & $1(3)$ & $1(3)$ \\
\hline & $28(100)$ & $28(100)$ \\
\hline
\end{tabular}

Table 2. Hormone receptor and HER2 status of the first and second lesions

\begin{tabular}{|c|c|c|}
\hline & First lesion & Second lesion \\
\hline & n. (\%) & n. (\%) \\
\hline ER-positive & $23(82)$ & $22(79)$ \\
\hline ER-negative & $5(18)$ & $6(21)$ \\
\hline & $28(100)$ & $28(100)$ \\
\hline & First lesion & Second lesion \\
\hline HER2-positive & n. (\%) & n. (\%) \\
\hline HER2-negative & $3(11)$ & $0(0)$ \\
\hline & $25(89)$ & $28(100)$ \\
\hline & $28(100)$ & $28(100)$ \\
\hline
\end{tabular}

Table 3. Histopathological diagnoses of the first and second lesions.

\begin{tabular}{|c|c|c|}
\hline & First lesion & Second lesion \\
\hline & n. (\%) & n. (\%) \\
\hline IDCa & $24(86)$ & $15(54)$ \\
\hline DCIS & $2(7)$ & $11(39)$ \\
\hline Mucinous Ca & $2(7)$ & $2(7)$ \\
\hline & $28(100)$ & $28(100)$ \\
\hline
\end{tabular}

Table 4. Positive-finding rate (sensitivity) within the second lesions.

\begin{tabular}{|c|c|c|c|}
\hline & MMG & US & $\begin{array}{c}\text { Contrast-enhanced } \\
\text { MRI }\end{array}$ \\
\hline & n. (\%) & n. (\%) & n. (\%) \\
\hline Positive & $13(46)$ & $18(64)$ & $28(100)$ \\
\hline Negative & $15(54)$ & $10(36)$ & $0(0)$ \\
\hline & $28(100)$ & $28(100)$ & $28(100)$ \\
\hline
\end{tabular}

while lesions in $13(46 \%)$ and 18 (64\%) patients were identified by MMG and ultrasound, respectively. In six of the 28 patients (21\%), contrastenhanced MRI visualized lesions that were undetectable by both MMG and ultrasonography. Breast MRI images of the aforementioned six patients are shown in Figure 1. The histopathological diagnoses and sizes of these tumors are listed in Table 5. Of these six patients, 5 patients $(83 \%)$ had IDCa and one (17\%) had apocrine carcinoma within the second lesion. No patient had DCIS. The median size of the invasive cancer was $2.5 \mathrm{~cm}$.

\section{Frequency of multiple breast lesions and family history status}

10 of the 28 patients (36\%) had multiple breast lesions. In 9 cases, two masses were found in the first lesion, and one case showed three masses in the first lesion.

Eight of the 28 patients (29\%) had a family history of breast cancer.

\section{Discussion}

It has been reported that contrast-enhanced MRI has a high diagnostic ability for mass lesions, with a sensitivity and a specificity of $99 \%$ and $89 \%$, respectively[7]. Many studies have also demonstrated the high accuracy of contrast-enhanced MRI in determining the extent of disease [4-6] as well as its effectiveness in detecting multiple lesions [4, 

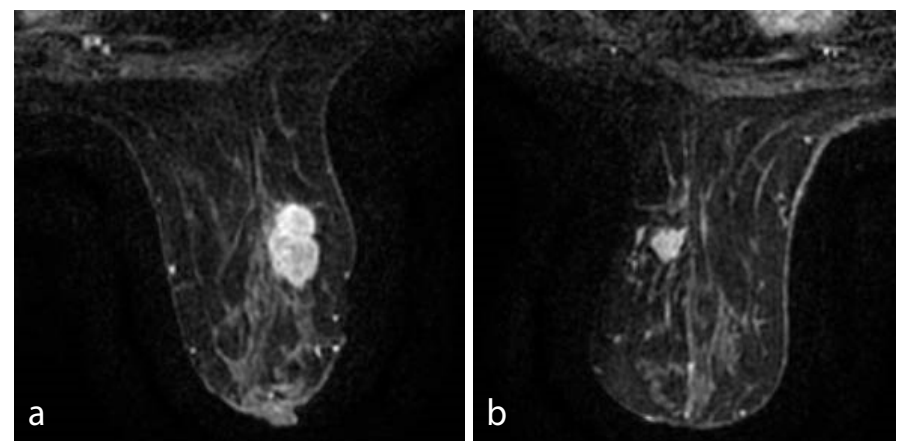

Figure 1. Contrast-enhanced MRI findings of the first and second lesions.

Case 1

a: The contrast-enhanced image shows a 23-mm mass suggestive of IDCa.

b: The contrast-enhanced scan displays an 8-mm nodule suggestive of IDCa. Pathology: 8-mm IDCa.
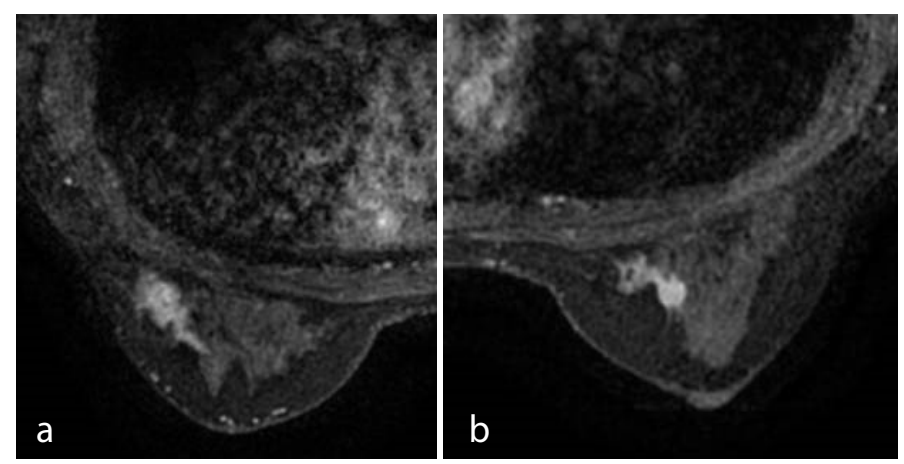

Case 2

a: Segmental enhancement suggestive of IDCa.

b: 16-mm mass with irregular margin suggestive of IDC. Pathology: 20-mm IDCa.
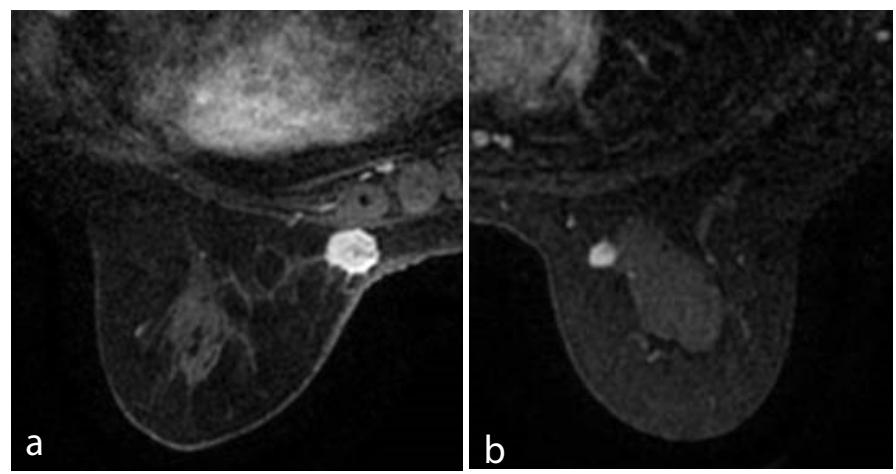

Case 3

a: $15-\mathrm{mm}$ mass with irregular margin suggestive of IDCa.

b: 6-mm nodule. Breast cancer could not be excluded. Pathology: 5-mm IDCa.

Table 5. Histopathological diagnoses of the 6 cases detected only by contrast-enhanced MRI

\begin{tabular}{|c|c|c|c|c|}
\hline & \multicolumn{2}{|c|}{ Histopathological diagnosis } & \multicolumn{2}{|c|}{ Tumor size } \\
\hline Case & First lesion & Second lesion & $\begin{array}{c}\text { First lesion } \\
(\mathbf{c m})\end{array}$ & $\begin{array}{c}\text { Second lesion } \\
(\mathbf{c m})\end{array}$ \\
\hline 1 & IDCa & IDCa & 2.8 & 0.8 \\
\hline 2 & IDCa & IDCa & 3.0 & 2.0 \\
\hline 3 & IDCa & IDCa & 1.4 & 0.5 \\
\hline 4 & IDCa & IDCa & 3.8 & 5.0 \\
\hline 5 & IDCa & IDCa & 1.5 & 2.4 \\
\hline 6 & IDCa & IDCa & 4.5 & 2 \\
\hline
\end{tabular}
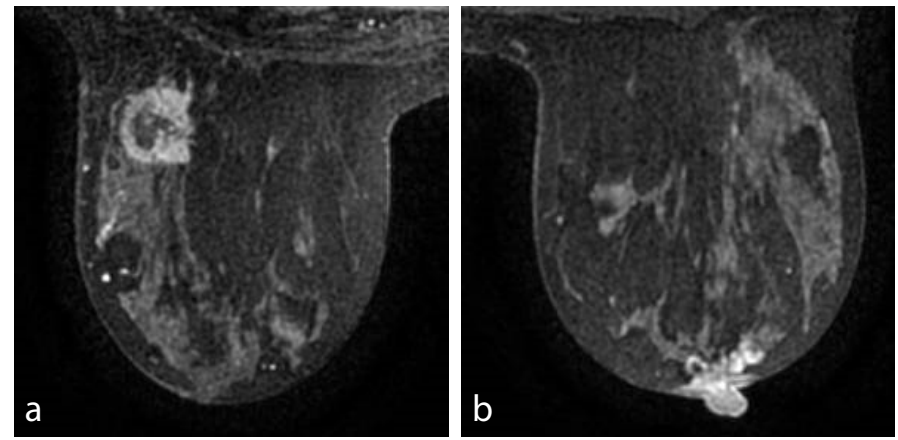

\section{Case 4}

a: 25-mm mass with irregular margin.

b: Stippled enhancements immediately behind the nipple are distributed in the upper area of the breast, suggestive of DCIS. Pathology: 50-mm IDCa.
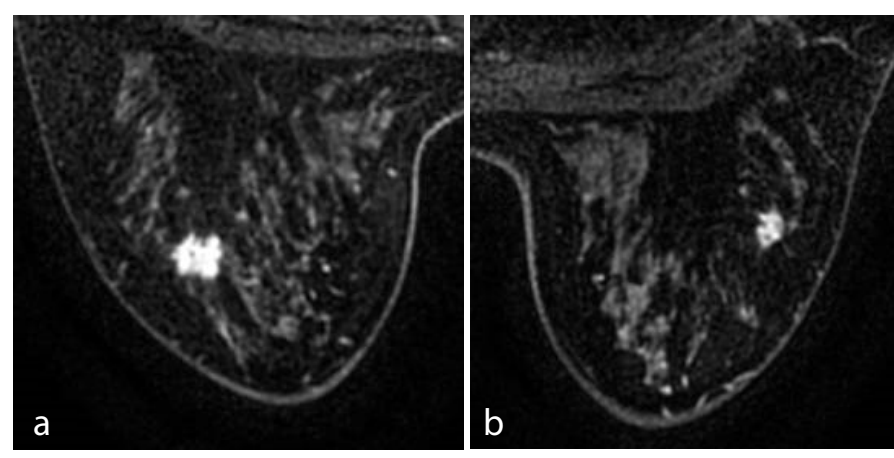

Case 5

a: 12-mm lobulated mass suggestive of IDCa.

b: 6-mm lobulated mass suggestive of IDCa. Pathology: 24-mm IDCa.
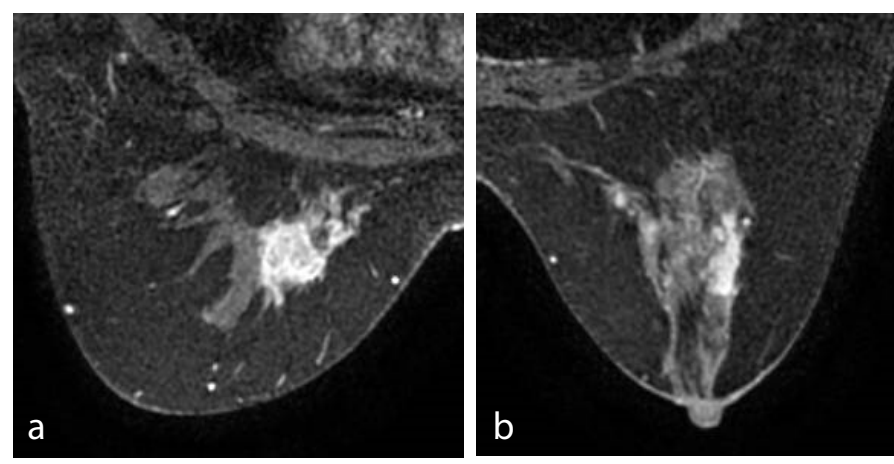

Case 6

a: 33-mm mass suggestive of IDCa or extensive intraductal carcinoma.

b: Stippled enhancement suggestive of DCIS. Pathology: 24-mm apocrine carcinoma.

6, 8-10] and contralateral breast cancer [11-15]. However, few studies have compared the utility of contrast-enhanced MRI with that of MMG or ultrasonography for detecting synchronous bilateral breast cancer.

In one study, Lehman et al. performed contrast-enhanced MRI in 969 patients who were diagnosed with unilateral breast cancer by MMG and clinical examination [11]. A biopsy was performed in 121 patients $(12.5 \%)$ on the basis of positive contrast-enhanced MRI findings in the contralateral breast. The biopsy confirmed malignancy in the contralateral breast in 30 patients (3.1\%). Thus, the sensitivity, specificity, and negative predictive value of contrast-enhanced MRI 
in identifying contralateral breast cancer were $91 \%, 88 \%$, and $99 \%$, respectively.

Our current study found that contrast-enhanced MRI has the highest sensitivity in detecting non-palpable contralateral breast cancers. The contralateral lesions in six of the 28 patients (21\%) were non-palpable and detected only by contrast-enhanced MRI. They were undetectable by MMG or by ultrasonography; thus, if contrast-enhanced MRI had not been performed in these patients, their contralateral lesions would not have been diagnosed. All of the six aforementioned cancers were found to be IDCa, with a median tumor size of $2.0 \mathrm{~cm}$ (range, 0.5-5.0 cm). Therefore, it was clinically important that we were able to identify these synchronous cancers by contrast-enhanced MRI and treat both tumor-bearing breasts simultaneously with a single surgery. The lesions of Cases 1 and 3 were $\leq 8 \mathrm{~mm}$ and were not visible by MMG or by ultrasound. These abnormalities that were detected by contrastenhanced MRI were subsequently confirmed by ultrasonography. In Cases 2 and 6, segmental enhancement was seen on MRI images, and a clear mass lesion was absent on both mammographic and ultrasonographic images; these imaging findings were indicative of DCIS. Pathologic examination, however, revealed that these patients had IDCa with tumor sizes of $2 \mathrm{~cm}$ and $2.4 \mathrm{~cm}$. IDCa was identified in Case 4 , which involved a $5.0-\mathrm{cm}$ mass immediately under the nipple that was difficult to detect by mammography and ultrasonography. Our findings therefore suggest that MRI is also effective in identifying large, hard-to-palpate IDCa.

Metachronous bilateral breast cancer was found in 42 of the 1465 breast cancer patients $(2.9 \%)$ who underwent surgery in our hospital between April 2006 and December 2012. The mean time interval to diagnosis of the second lesion was 12 years (range, 2-32 years). No cases of contralateral breast cancer were diagnosed within two years of initial diagnosis at our hospital, which may also indicate the applicability of contrast-enhanced MRI in detecting contralateral breast cancer.

There has recently been a heightened interest in hereditary breast cancer. One of its characteristics is bilateral breast cancer. Although it has been noted worldwide that an increasing number of institutions offer contralateral prophylactic mastectomy (CPM), guidelines issued by the National Comprehensive Cancer Network (NCCN) discourage the use of CPM, and provide ambiguous recommendations for its use. Early detection of bilateral breast cancer by performing preoperative contrastenhanced MRI would enable better surgical decision making compared to those made solely by MMG and ultrasonography-driven information.

\section{Conclusion}

Contrast-enhanced MRI is a highly sensitive and therefore valuable tool for identifying non-palpable breast cancers. It is especially useful in the detection of small contralateral lesions and lesions immediately under the nipple of the contralateral breast.

\section{Conflict of interest}

The authors declare that they have no conflict of interest.

\section{References}

1. Heron DE, Komarnicky LT, Hyslop T, Schwartz GF, Mansfield CM (2000) Bilateral breast carcinoma: risk factors and outcomes for patients with synchronous and metachronous disease. Cancer 88: 2739-2750. [Crossref]

2. Chen JJ, Wang Y, Xue JY, Chen Y, Chen YL, et al. (2014) A clinicopathological study of early-stage synchronous bilateral breast cancer: a retrospective evaluation and prospective validation of potential risk factors. PLoS One 9: e95185.

3. Shi YX, Xia Q, Peng RJ, Yuan ZY, Wang SS, et al. (2012) Comparison of clinicopathological characteristics and prognoses between bilateral and unilateral breast cancer. J Cancer Res Clin Oncol 138: 705-714. [Crossref]

4. Boetes C, Mus RD, Holland R, Barentsz JO, Strijk SP, et al. (1995) Breast tumors comparative accuracy of MR imaging relative to mammography and US for demonstrating extent. Radiology 197: 743-747. [Crossref]

5. Amano G, Ohuchi N, Ishibashi T, Ishida T, Amari M, et al. (2000) Correlation of three-dimensional magnetic resonance imaging with precise histopathological map concerning carcinoma extension in the breast. Breast Cancer Res Treat 60: 43-55. [Crossref]

6. Esserman L, Hylton N, Yassa L, Barclay J, Frankel S, et al. (1999) Utility of magnetic resonance imaging in the management of breast cancer: evidence for improved preoperative staging. J Clin Oncol 17: 110-119. [Crossref]

7. Tozaki M, Igarashi T and Fukuda K (2006) Positive and negative predictive values of BI-RADS-MRI descriptors for focal breast masses. Magn Reson Med Sci 5: 7-15. [Crossref]

8. Harms SE, Flamig DP, Hesley KL, Meiches MD, Jensen RA, et al. (1993) MR imaging of the breast with rotating delivery of excitation off resonance: clinical experience with pathologic correlation. Radiology 187: 493-501. [Crossref]

9. Orel SG, Schnall MD, Powell CM, Hochman MG, Solin LJ, et al. (1995) Staging of suspected breast cancer: effect of MR imaging and MR-guided biopsy. Radiology 196: 115-122. [Crossref]

10. Fischer U, Kopka L and Grabbe E (1999) Breast carcinoma: effect of preoperative contrast-enhanced MR imaging on the therapeutic approach. Radiology 213: 881-888. [Crossref]

11. Lehman CD, Gatsonis C, Kuhl CK, Hendrick RE, Pisano ED, et al. (2007) MRI evaluation of the contralateral breast in women with recently diagnosed breast cancer. N Engl J Med 356: 1295-1303. [Crossref]

12. Lee SG, Orel SG, Woo IJ, Cruz-Jove E, Putt ME, et al. (2003) MR imaging screening of the contralateral breast in patients with newly diagnosed breast cancer: preliminary results. Radiology 226: 773-778.

13. Liberman L, Morris EA, Kim CM, Kaplan JB, Abramson AF, et al. (2003) MR imaging findings in the contralateral breast of women with recently diagnosed breast cancer AJR Am J Roentgenol 180: 333-341.

14. Viehweg P, Rotter K, Laniado M, Lampe D, Buchmann J, et al. (2004) MR imaging of the contralateral breast in patients after breast-conserving therapy. Eur Radiol 14: 402-408. [Crossref]

15. Friedman PD, Swaminathan SV, Herman K, Kalisher L (2006) Breast MRI: the importance of bilateral imaging. AJR Am J Roentgenol 187: 345-349. [Crossref]

Copyright: (C2017 Kosaka Y. This is an open-access article distributed under the terms of the Creative Commons Attribution License, which permits unrestricted use, distribution, and reproduction in any medium, provided the original author and source are credited. 